\title{
Grand unification and intermediate scale supersymmetry
}

\author{
Lawrence J. Hall and Yasunori Nomura \\ Berkeley Center for Theoretical Physics, Department of Physics, \\ University of California, Berkeley, CA 94720, U.S.A. \\ Theoretical Physics Group, Lawrence Berkeley National Laboratory, \\ Berkeley, CA 94720, U.S.A. \\ E-mail: ljhall@lbl.gov, ynomura@berkeley.edu
}

ABSTRACT: With minimal field content and for an interesting range of the supersymmetric Higgs mixing parameter, $0.5 \lesssim \tan ^{2} \beta \lesssim 2$, the superpartner mass scale, $\tilde{m}$, is found to be at the intermediate scale, $\sim 10^{10 \pm 1} \mathrm{GeV}$, near where the Standard Model Higgs quartic coupling passes through zero. For any $4 \mathrm{~d}$ supersymmetric grand unified symmetry spontaneously broken by a vacuum expectation value $\langle\Sigma\rangle$, if superpotential interactions for $\Sigma$ are forbidden e.g. by $R$ symmetries, the uneaten color octet, $\Sigma_{8}$, and weak triplet, $\Sigma_{3}$, have masses of order $\tilde{m}$. The combination of superpartner and $\Sigma_{8,3}$ states leads to successful gauge coupling unification, removing the disastrously high proton decay rate of minimal Standard Model unification. Proton decay could be seen in future experiments if $\tilde{m} \sim 10^{11} \mathrm{GeV}$, but not if it is lower. If the reheating temperature after inflation, $T_{R}$, is less than $\tilde{m}$ dark matter may be axions. If $T_{R}>\tilde{m}$, thermal LSP dark matter may lead to the environmental selection of a TeV-scale LSP, either wino or Higgsino, which could comprise all or just one component of dark matter. In the Higgsino case, the dark matter is found to behave inelastically in direct detection experiments, and gauge coupling unification occurs accurately without the need of any threshold corrections.

KEYWords: Supersymmetry Breaking, Beyond Standard Model, GUT

ARXIV EPRINT: 1312.6695 


\section{Contents}

1 Introduction 1

$2 \tilde{m}$ from the zero of the Higgs quartic $\quad 3$

3 Minimal unified theory with ISS 4

4 Supersymmetry breaking and dark matter $\quad 6$

4.1 Pure intermediate scale supersymmetry 8

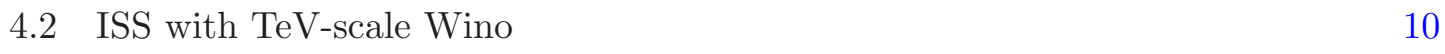

4.3 ISS with TeV-scale Higgsino 11

\section{Introduction}

The discovery of a Higgs boson with mass near $125 \mathrm{GeV}$ not only completes the Standard Model (SM), but allows the SM to provide a perturbative description of nature up to extremely high energies. Such a "desert" hypothesis was first suggested in the context of the unification of the SM into an SU(5) grand unified theory $[1,2]$. In the following four decades, data consistently kept open this possibility, even while the theoretical emphasis was on extended models of particle physics at the weak scale. Precision measurements of gauge couplings were broadly consistent with their perturbative unification at very high energies, neutrino masses were consistent with dimension- 5 operators suppressed by a large mass near the unified scale, and all quark and charged lepton masses were found to be sufficiently light that the corresponding Yukawa couplings remained perturbative to unified energies. A Higgs boson lighter than about $110 \mathrm{GeV}$ would exclude such a large energy desert, as the electroweak vacuum would be unstable with a lifetime much less than $10^{10}$ years; and a Higgs boson heavier than about $190 \mathrm{GeV}$ would have led to the Higgs quartic coupling becoming non-perturbative well below the unified mass, heralding a new scale of nonperturbative physics. Perhaps even more remarkable than the discovery of a Higgs boson in this high energy desert window of $110-190 \mathrm{GeV}$, is the absence of a single discovery of physics beyond the SM during these four decades: an extension of the SM at the weak scale has had ample opportunity to reveal itself in rare flavor and $C P$ violating processes, at a succession of collider experiments, culminating in the negative results at the LHC so far, and in both direct and indirect searches for dark matter in our galaxy.

The most glaring shortcoming of the desert hypothesis is that the weak scale is highly unnatural, requiring many orders of magnitude of fine-tuning. Indeed, this was the principle driver behind theoretical efforts at extending the SM at the weak scale and experimental programs for beyond SM physics at LEP, Tevatron, and the LHC. Yet, as with the cosmological constant [3-5], this fine-tuning may result from environmental selection on a 
multiverse. While the physical origin of this selection is uncertain, one possibility is the requirement of complex nuclei $[6,7]$.

While experiment indicates a broad picture of perturbative unification of the SM, there are difficulties with the simplest implementation. First and foremost, the precision of gauge coupling unification is limited: a $6 \%$ correction at the unified scale is required and, furthermore, to be consistent with limits on proton stability this correction must raise the superheavy gauge boson mass $M_{X}$ from about $10^{14} \mathrm{GeV}$ to above $6 \times 10^{15} \mathrm{GeV}$. Secondly, the mass ratios of down-type quarks to charged leptons do not agree with the simplest $\mathrm{SU}(5)$ expectations.

In this paper we seek an origin for precision gauge coupling unification that is linked to the value of the Higgs boson mass. The Higgs quartic coupling, when evolved to high energies, becomes negative and is small. Is there a simple physical understanding of this behavior? One possibility is that the quartic scans in the landscape with a distribution favoring negative values, and that our universe is typical among those with a sufficiently long-lived electroweak vacuum to allow observers [8]. In this paper we pursue an alternative idea - the underlying theory of nature is supersymmetric and the scale of the SM superpartners, $\tilde{m}$, is close to the scale at which the Higgs quartic coupling $\lambda$ passes through zero:

$$
\tilde{m} \sim \mu_{c} \quad \text { with } \quad \lambda\left(\mu_{c}\right)=0 .
$$

Since the current experimental data indicates $\mu_{c} \simeq 10^{10}-10^{12} \mathrm{GeV}[9,10]$, this leads to the picture of Intermediate Scale Supersymmetry (ISS).

Values of $\tilde{m}$ as high as the unified scale are possible if one takes into account the uncertainty of the top quark mass determination at a level of $2-3 \sigma$. Indeed, we previously predicted that in High Scale Supersymmetry, with the SM valid up to unified scales where it becomes supersymmetric, the Higgs boson mass is (128 \pm 3$) \mathrm{GeV}$ for $\tan \beta=1$ [11]; see also [12-14] for more recent analyses. This prediction was found to be highly insensitive to the scale of $\tilde{m}$, dropping by only $1 \mathrm{GeV}$ as the scale is reduced from $10^{14} \mathrm{GeV}$ to $10^{12} \mathrm{GeV}$. Furthermore, $\tan \beta$ of unity can result from an approximate discrete symmetry that interchanges the two Higgs doublets.

Here we take the view that the supersymmetric boundary condition in eq. (1.1) occurs substantially below the unified scale, allowing us to connect predictions for the Higgs boson mass with those for precision gauge coupling unification and proton decay. Thus we assume that the unified symmetry is broken at scale $M_{X}$ to the Minimal Supersymmetric Standard Model (MSSM), with supersymmetry broken at $\tilde{m} \ll M_{X}$. In any such unified scheme, the superheavy gauge particles acquire mass by eating part of an SU(5) adjoint multiplet $\Sigma$. The remaining states of $\Sigma$, with mass $M_{\Sigma}$, are therefore in a $\mathrm{SU}(5)$-split representation and contribute to gauge coupling unification. One frequently assumes that $M_{\Sigma} \sim M_{X}$, but this need not be the case. A symmetry may forbid a $\Sigma$ mass term from appearing in the superpotential, while allowing it to arise from supersymmetry breaking, so that $M_{\Sigma} \sim \tilde{m}$. This is very much analogous to $\mu \sim \tilde{m}$ in the MSSM. The combination of gauginos and $\Sigma$ improves gauge coupling unification, and allows $M_{X}>6 \times 10^{15} \mathrm{GeV}$ for $\tilde{m} \lesssim 2 \times 10^{11} \mathrm{GeV}$, making it consistent with the constraints from proton decay [15] without resorting to a special mechanism at the unified scale. 
The ISS framework discussed in this paper allows for three different manifestations, depending on how the environmental selection effect works in the multiverse. The first, in some sense the simplest, possibility is that the masses of all the superpartners are within a couple of orders of magnitude at $\tilde{m}$. The other two cases are that the wino and Higgsino masses, respectively, are brought down to the $\mathrm{TeV}$ scale so that they comprise (a part of) the dark matter; all the other superpartner masses stay around $\tilde{m}$. In the case of Higgsino dark matter, splitting between the two neutral components are of $O(100 \mathrm{keV})$ naturally yielding inelastic dark matter [16]. Precision gauge coupling unification may be analyzed in each case. In the case of TeV-scale Higgsinos the ratios of the observed gauge couplings are reproduced from the unification condition at the level of the MSSM without extra threshold corrections, while in the other two cases threshold corrections from a dimension5 operator, of natural size, are required at the unified scale. In either of the three cases, however, the mass of the superheavy gauge bosons can be determined with some precision, giving relatively tight implications for the proton decay rate.

The organization of the rest of the paper is as follows. In section 2, we discuss the condition in eq. (1.1), motivating ISS. In section 3, we introduce our minimal unified theory with ISS in which $M_{\Sigma} \sim \tilde{m}$ arises in a simple manner. In section 4 , we discuss supersymmetry breaking and environmental selection for dark matter. We consider three different manifestations of our framework: pure ISS, ISS with TeV-scale wino, and ISS with $\mathrm{TeV}$-scale Higgsino. Gauge coupling unification and proton decay are discussed in each case.

\section{$2 \tilde{m}$ from the zero of the Higgs quartic}

We take the theory below the unified scale to be the MSSM with the mass squared matrix for the two Higgs doublets given by

$$
\mathcal{M}_{\mathrm{H}}^{2}=\left(\begin{array}{cc}
|\mu|^{2}+m_{H_{u}}^{2} & \mu B \\
\mu B & |\mu|^{2}+m_{H_{d}}^{2}
\end{array}\right),
$$

where the soft supersymmetry breaking parameters $m_{H_{u}, H_{d}}^{2}$ and $B$ are governed by the scale $\tilde{m}$. As we will see later, we consider that the unified theory above the unified scale is the minimal supersymmetric $\mathrm{SU}(5)$ model $[17,18]$ or its simple extensions, e.g. to $\mathrm{SO}(10)$, so that the supersymmetric mass parameter $\mu$ arises from a cancellation of terms of order the unified scale. Note that such a cancellation occurs as a consequence of environmental selection of having the correct electroweak symmetry breaking, which requires $|\mu| \lesssim \tilde{m}$.

The theory below $\tilde{m}$ is the SM, possibly with a few supersymmetric partners required by other environmental requirements. (We will later consider the case in which the Higgsinos or winos are at a $\mathrm{TeV}$ scale due to environmental selection associated with dark matter.) This requires the determinant of the matrix $\mathcal{M}_{\mathrm{H}}^{2}$ to be extremely small compared with its natural size $\sim \tilde{m}^{4}$, forced by the environmental condition of electroweak symmetry breaking. Writing the SM Higgs potential as

$$
V(h)=-m^{2} h^{\dagger} h+\frac{\lambda}{4}\left(h^{\dagger} h\right)^{2},
$$


the quartic coupling at the scale $\tilde{m}$ is dominated by the tree-level result

$$
\lambda(\tilde{m})=\frac{g^{2}+g^{\prime 2}}{2} \cos ^{2} 2 \beta \quad \text { with } \quad \tan ^{2} \beta=\frac{|\mu|^{2}+m_{H_{d}}^{2}}{|\mu|^{2}+m_{H_{u}}^{2}}
$$

where the $\mathrm{SU}(2) \times \mathrm{U}(1)$ gauge couplings $g, g^{\prime}$ and the parameters $\mu, m_{H_{u}}^{2}, m_{H_{d}}^{2}$ are all evaluated at scale $\tilde{m}$.

A key point is that there is a large region of parameter space in which $\lambda(\tilde{m})$ is very small, so that eq. (1.1) can be used to obtain the correct order of magnitude of $\tilde{m}$. To begin with, suppose that an approximate symmetry leads to $m_{H_{u}, H_{d}}^{2}$ being approximately equal, allowing an expansion $\tan ^{2} \beta=1+\epsilon$, with $\epsilon \ll 1$. One discovers that $\lambda(\tilde{m})$ is remarkably small, arising only at quadratic order

$$
\lambda(\tilde{m}) \simeq 0.06 \epsilon^{2}+O\left(\epsilon^{3}\right),
$$

where the coefficient reflects the values of the gauge couplings at the scale $\sim 10^{10} \mathrm{GeV}$, where the SM Higgs quartic is near zero. Indeed, in the SM the Higgs quartic coupling vanishes at $\mu_{c} \simeq 10^{10}-10^{12} \mathrm{GeV}$, but providing $\lambda(\tilde{m})<0.02, \tilde{m}$ is less than an order of magnitude below $\mu_{c}$. Comparing with eq. (2.4) we see that $\epsilon$ need not be very small for this to happen. Thus we can take the condition of vanishing $\lambda$ to determine the correct order of magnitude for $\tilde{m}$ provided $\lambda(\tilde{m})<0.02$, which translates into

$$
0.55 \lesssim \tan ^{2} \beta=\frac{|\mu|^{2}+m_{H_{d}}^{2}}{|\mu|^{2}+m_{H_{u}}^{2}} \lesssim 1.8,
$$

where we have used the full expression in eq. (2.3), instead of eq. (2.4). This does not require near degeneracy between $m_{H_{u}, H_{d}}^{2}$; indeed they can differ by much more than is generated by renormalization group scaling from the unified scale using the top Yukawa coupling.

\section{Minimal unified theory with ISS}

The simple group of lowest rank that allows unification of the known gauge forces is $\mathrm{SU}(5)$ [1]. With supersymmetry restored at the intermediate scale, $\tilde{m} \sim \mu_{c} \simeq$ $10^{10}-10^{12} \mathrm{GeV}$, we seek a supersymmetric unified theory, and the minimal field content is well known [17, 18]: Higgs chiral superfields $\Sigma(\mathbf{2 4})$ to break $\mathrm{SU}(5)$ and $H(\mathbf{5}), \bar{H}\left(\mathbf{5}^{*}\right)$ to break the electroweak interaction, together with matter chiral superfields $T(\mathbf{1 0})$ and $\bar{F}\left(\mathbf{5}^{*}\right)$ containing quarks and leptons. Below, we assume that $R$ parity is conserved. We omit the generation index for quarks and leptons throughout.

There are four possible interactions in the superpotential involving the Higgs multiplets that have dimension 4 or less: two mass terms, $[\bar{H} H]_{\theta^{2}}$ and $\left[\Sigma^{2}\right]_{\theta^{2}}$, and two trilinear interactions, $[H \Sigma \bar{H}]_{\theta^{2}}$ and $\left[\Sigma^{3}\right]_{\theta^{2}}$. This should be compared to the MSSM, where there is a single such possible Higgs interaction in the superpotential $\left[\mu H_{u} H_{d}\right]_{\theta^{2}}$. The phenomenology of weak scale supersymmetry requires that $\mu \sim \tilde{m} \sim \mathrm{TeV}$, the well known " $\mu$ " problem of the MSSM. With high scale mediation of supersymmetry breaking, it is easy to solve this problem by forbidding such a superpotential term and instead allowing a bilinear interaction between the two Higgs field in the Kähler potential, $\left[H_{u} H_{d}\right]_{\theta^{4}}$, since in supergravity 
this leads to an effective term in the superpotential with coefficient proportional to the gravitino mass, $\mu=c m_{3 / 2}$, where $c$ is a constant of $O(1)[19,20]$.

With ISS we can ask whether either of the Higgs mass terms, $H \bar{H}$ and $\Sigma^{2}$, might similarly appear in the Kähler potential rather than in the superpotential. For $H \bar{H}$ we choose to put it in the superpotential, which makes the colored triplets in these multiplets have a mass of order the unified scale. The superpotential of the minimal ISS theory then contains

$$
W=m_{H} H \bar{H}+\lambda_{H} H \Sigma \bar{H}+y_{U} T T H+y_{D} T \bar{F} \bar{H} .
$$

If the theory possesses an $R$ symmetry in the supersymmetric limit, then the presence of the first two terms forbids $\Sigma^{2}$ and $\Sigma^{3}$ from appearing in the superpotential $W$, while allowing them in the Kähler potential

$$
K \supset \frac{c_{2}}{2} \Sigma^{2}+\frac{c_{3}}{3 \Lambda} \Sigma^{3}
$$

where $c_{2,3}$ are dimensionless couplings of order unity, while $\Lambda$ is the UV cutoff of the unified theory, which we expect to be within an order of magnitude from the reduced Planck scale $M_{\mathrm{Pl}}$. As in the case of $H_{u} H_{d}$ in the MSSM, supergravity then generates an effective superpotential

$$
W_{\text {eff }}=\frac{m_{\Sigma}}{2} \Sigma^{2}+\frac{\lambda_{\Sigma}}{3} \Sigma^{3}
$$

where $m_{\Sigma}=c_{2} m_{3 / 2}^{*}$ and $\lambda_{\Sigma}=c_{3} m_{3 / 2}^{*} / \Lambda$. In this paper, we assume that the mediation scale of supersymmetry breaking $M_{*}$ is high, $M_{*} \sim \Lambda \sim M_{\mathrm{Pl}}$. This implies that $m_{3 / 2}=F_{X} / \sqrt{3} M_{\mathrm{Pl}} \sim \tilde{m} \approx O\left(F_{X} / M_{*}\right)$, where $F_{X}$ is the $F$-term vacuum expectation value (VEV) of supersymmetry breaking field $X$. The key point is that the parameters appearing in eq. (3.3) are then $m_{\Sigma} \sim \tilde{m}$ and $\lambda_{\Sigma} \sim \tilde{m} / \Lambda$, which are both highly suppressed compared to conventional supersymmetric unified theories and are correlated with the scale of superpartner masses $\tilde{m}$. An alternative way of obtaining the effective superpotential of eq. (3.3), which is available if the supersymmetry breaking field $X$ is singlet, is to have the Kähler potential terms

$$
K \supset \frac{c_{2}^{\prime}}{2 M_{*}} X^{\dagger} \Sigma^{2}+\frac{c_{3}^{\prime}}{3 \Lambda M_{*}} X^{\dagger} \Sigma^{3} .
$$

In this case $m_{\Sigma} \sim \tilde{m}$ and $\lambda_{\Sigma} \sim \tilde{m} / \Lambda$, and these parameters are directly related with the superpartner mass scale $\tilde{m}$.

Assuming that the interactions in eq. (3.3) dominate over the soft supersymmetry breaking interactions for the scalar component $\phi_{\Sigma}$, which may not be an accurate approximation but is sufficient for the purpose of discussion here, we find a vacuum that breaks $\mathrm{SU}(5)$ to $\mathrm{SU}(3) \times \mathrm{SU}(2) \times \mathrm{U}(1)$

$$
\langle\Sigma\rangle=\frac{V}{\sqrt{60}}\left(\begin{array}{ccccc}
2 & 0 & 0 & 0 & 0 \\
0 & 2 & 0 & 0 & 0 \\
0 & 0 & 2 & 0 & 0 \\
0 & 0 & 0 & -3 & 0 \\
0 & 0 & 0 & 0 & -3
\end{array}\right),
$$


with $V=\sqrt{60} c_{2} \Lambda / c_{3}$ (or $c_{2,3} \rightarrow c_{2,3}^{\prime}$ if the relevant operators are as in eq. (3.4), rather than eq. (3.2)). If $c_{2} / c_{3} \sim 1$, the $\mathrm{VEV}$ of $\Sigma$ is so close to the UV cutoff that it is not reliably computed. To avoid this we take $c_{2}$ to be sufficiently smaller than $c_{3}$, which is equivalent to taking $m_{\Sigma}$ to be sufficiently smaller than $\Lambda$ in conventional unified theories.

Adding soft supersymmetry breaking interactions $\tilde{m}_{\Sigma}^{2} \phi_{\Sigma}^{\dagger} \phi_{\Sigma}-\left\{\left(B m_{\Sigma} / 2\right) \phi_{\Sigma}^{2}+\right.$ $\left(A \lambda_{\Sigma} / 3\right) \phi_{\Sigma}^{3}+$ h.c. $\}$, the precise locations of the minima of the potential will shift, leading to the naive expectation that $F_{\Sigma} \sim \tilde{m} \Lambda$. Inserting this into the superpotential interaction $H \Sigma \bar{H}$ then yields $\mu B \sim \tilde{m} \Lambda$, so that a light Higgs doublet for electroweak symmetry breaking then requires $\mu \sim \sqrt{\tilde{m} \Lambda} \gg \tilde{m}$. However, such large supersymmetry breaking in the Higgs sector is forbidden because a negative mass-squared for the top squark arises at 1 loop order, leading to a very large spontaneous breaking of the color group. The environmental requirement of an unbroken color symmetry forces a cancellation amongst terms so that $F_{\Sigma} \lesssim 4 \pi^{2} \tilde{m}^{2}$. This makes it probable that $\mu^{2}$ and $\mu B$ are an order of magnitude larger than $m_{H_{u, d}}^{2}$, leading to a Higgs quartic coupling of eq. (2.4) with $\epsilon \sim 0.1$.

\section{Supersymmetry breaking and dark matter}

Supersymmetry breaking is described by a spurion field $X$ that has a non-zero $F$ component, $F_{X}$. This supersymmetry breaking is transmitted to the MSSM sector at a high mediation scale $M_{*} \sim M_{\mathrm{Pl}}$, so that $\tilde{m}=F_{X} / M_{*}$ is not much larger than the gravitino mass $m_{3 / 2}$. In general, we include all the supersymmetry breaking operators in the Kähler potential

$$
\begin{aligned}
\mathcal{L}_{\mathrm{SB}} \sim\left[\left(\frac{X^{\dagger} X}{M_{*}^{2}}+\frac{X+\text { h.c. }}{M_{*}}\right)\left(T^{\dagger} T+\bar{F}^{\dagger} \bar{F}+H^{\dagger} H+\bar{H}^{\dagger} \bar{H}+\Sigma^{\dagger} \Sigma\right)\right]_{\theta^{4}} \\
+\left[\left\{\frac{X^{\dagger}}{M_{*}}\left(\Sigma^{2}+\Sigma^{3}+\cdots\right)+\text { h.c. }\right\}+\frac{X^{\dagger} X}{M_{*}^{2}}\left(\Sigma^{2}+\Sigma^{3}+\cdots\right)\right]_{\theta^{4}},
\end{aligned}
$$

where $\{T, \bar{F}\}$ and $\{H, \bar{H}, \Sigma\}$ are the matter and Higgs chiral superfields, and the terms associated with $H \bar{H}$ are assumed to be forbidden by the $R$ symmetry discussed below eq. (3.1). Note that each operator has an unknown coefficient of order unity that is not displayed. We also allow the operator in the gauge kinetic function

$$
\mathcal{L}_{\mathrm{SB}}^{\lambda} \sim\left[\frac{X}{M_{*}} \mathcal{W}^{\alpha} \mathcal{W}_{\alpha}\right]_{\theta^{2}}+\text { h.c. }
$$

where $\mathcal{W}^{\alpha}$ is the gauge field-strength superfield, but we forbid superpotential interactions coupling $X$ directly to matter and Higgs fields. If $X$ is not neutral, some of the terms described above are forbidden. For example, if it is charged under some symmetry, under which all the SU(5) fields are neutral, then the terms linear in $X$ are all forbidden.

A significant model dependence for the superpartner spectrum may arise from the values of the Higgsino mass, $\mu$, and the gaugino masses $\tilde{m}_{i}$ for $i=\mathrm{SU}(3), \mathrm{SU}(2), \mathrm{U}(1)$. The $\mu$ parameter arises from the first two operators of eq. (3.1) and from supersymmetry breaking

$$
\mu=m_{H}-\frac{3}{\sqrt{60}} \lambda_{H} V+O(\tilde{m})
$$


Fine-tuning between the first two terms is required so that $\mu$ is of order $\tilde{m}$ to ensure that the matrix of eq. (2.1) has a small eigenvalue, allowing the scale of weak interactions to be far below $\tilde{m}$. The gaugino masses, in general, have four contributions

$$
\tilde{m}_{i}=\tilde{m}_{i}^{0}+\tilde{m}_{i}^{\mathrm{AM}}+\tilde{m}_{i}^{\tilde{h}}+\tilde{m}_{i}^{\Sigma} .
$$

The first term arises from eq. (4.2) and occurs only if $X$ is neutral under all symmetries. For $M_{*}>V$, this gives the contribution $\tilde{m}_{i} \approx \tilde{m} \alpha_{i}\left(\mu=\mu_{c}\right) / \alpha_{5}\left(\mu=M_{*}\right)$, where $\alpha_{i}=g_{i}^{2} / 4 \pi$ are the gauge field strength, with $\alpha_{5}$ being their unified value. The remaining terms occur at one loop and are relevant only if $X$ carries a charge so that the leading term is absent. The second term arises from anomaly mediation $[21,22]$ giving $\tilde{m}_{i}^{\mathrm{AM}}=b_{i} g_{i}^{2} m_{3 / 2} / 16 \pi^{2}$, where $\left(b_{1}, b_{2}, b_{3}\right)=(33 / 5,1,-3)$ are the beta-function coefficients, and we have taken the phase convention that $\tilde{m}_{1,2}$ are real and positive. The third contribution is relevant only if the Higgsinos are heavier than the gauginos, as it arises from integrating out the Higgsinos, and contributes only to $\tilde{m}_{1,2}$. The fourth term arises from integrating out the uneaten states of $\Sigma$, and contributes only to $\tilde{m}_{2,3}$.

We assume that $R$-parity is unbroken, so that the lightest supersymmetric particle (LSP) is stable and contributes to the dark matter if it is produced cosmologically. We further assume environmental constraints that strongly disfavor observers in universes with much more dark matter than our own, as argued, e.g., in [23, 24]. This yields a large environmentally forbidden window in $m_{\mathrm{LSP}}[25]$

$$
c_{-} \mathrm{TeV}<m_{\mathrm{LSP}}<c_{+} T_{R}
$$

where $c_{-}$depends on the LSP annihilation rate and is order unity while $c_{+} \sim 10-30$, depending on the size of the reheating temperature after inflation, $T_{R}$. If $m_{\mathrm{LSP}}>c_{-} \mathrm{TeV}$ the LSP is overproduced at freezeout, unless $m_{\mathrm{LSP}}>c_{+} T_{R}$ so that it never gets close to thermal equilibrium. Taking $\tilde{m}$ to be fixed around $\mu_{c} \simeq 10^{10}-10^{12} \mathrm{GeV}$, as determined by the $125 \mathrm{GeV}$ Higgs boson mass, it is critical whether the resulting value of $m_{\mathrm{LSP}}$ is above or below $c_{+} T_{R}$.

- If $m_{\mathrm{LSP}}<c_{+} T_{R}$, fine-tuning between contributions to $m_{\mathrm{LSP}}$ is required to force $m_{\mathrm{LSP}}<c_{-} \mathrm{TeV}$. Dark matter will then have a sizable LSP component, although there could be other sizable components such as axions.

- If $m_{\mathrm{LSP}} \geq c_{+} T_{R}$, there are two possibilities. If there is non-LSP dark matter, such as axions, then an environmental selection for dark matter does not force any fine-tuning in the superpartner masses (beyond that already required in $\mu$ to allow electroweak symmetry breaking). The LSP contribution to dark matter will be negligible, and no superpartners are expected in the $\mathrm{TeV}$ domain. On the other hand, if there is no axion or other source for dark matter, then an environmental requirement for significant dark matter will force $m_{\mathrm{LSP}} \sim c_{+} T_{R}$, so that dark matter is produced at reheating from collisions involving the high energy tail of thermal distributions. In this case, the LSP will contribute all of the dark matter. No superpartners, however, are expected in the TeV domain, since the LSP is rather heavy of mass $\sim c_{+} T_{R}$. 

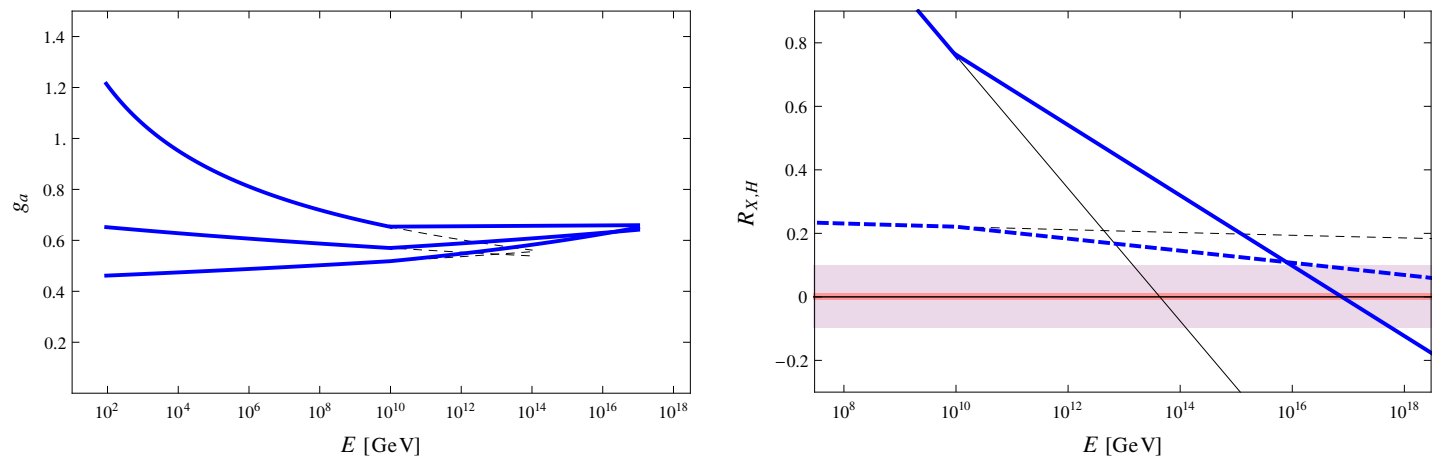

Figure 1. Pure intermediate scale supersymmetry breaking. Left panel: evolution of gauge couplings in ISS (solid, blue) and in the SM (dashed, black). Right panel: evolution of $R_{X}$ (solid) and $R_{H}$ (dashed) for ISS (thick, blue) and the SM (thin, black). Without corrections from dimension 5 and 6 operators, $M_{X}$ and $M_{H}$ are the scales at which $R_{X}$ and $R_{H}$ vanish, respectively. Including the dimension 5 operator, allowed values of $M_{H}$ occur when the dashed thick (blue) line lies inside the pale (red) shaded region. Including the dimension 6 operators, allowed values of $M_{X}$ occur when the solid thick (blue) line lies inside the dark (red) shaded region. Both shaded (red) bands have been drawn for $V / \Lambda=0.1$.

Which of these scenarios is realized is model dependent. Below we consider three representative cases. We first consider in section 4.1 a class of models that requires no fine-tuning of $m_{\mathrm{LSP}}$ : the first case discussed in the second bullet point given above. In this case, the LSP is extremely heavy and its nature is rather unimportant. We then consider two models of the type described in the first bullet point, in which fine-tuning of the LSP mass is required. Note that whenever the LSP mass is environmentally forced to be much less than its typical value, the LSP will be a fermion, since the tuning in $m_{\mathrm{LSP}}$ is linear for a fermion and quadratic for a boson. Hence in one of these models the LSP is a wino and in the other a Higgsino, which we will discuss in sections 4.2 and 4.3, respectively.

\subsection{Pure intermediate scale supersymmetry}

A particularly simple possibility is that all the superpartner masses are within one or two orders of magnitude of the scale $\tilde{m}$ determined by eq. (1.1). This would occur, for example, if all the supersymmetry breaking operators in eqs. (4.1) and (4.2) occur with unsuppressed coefficients. In this case we take the reheating temperature after inflation to be sufficiently low that none of the superpartners have a significant cosmological abundance. This requires baryogenesis at a relatively low temperature which could result, for larger values of $\tilde{m}$, from thermal leptogenesis. Dark matter would be non-supersymmetric, for example axions.

The unification of gauge couplings for this class of models is illustrated by the solid blue lines in the left panel of figure 1. The masses of all superpartners and uneaten components of $\Sigma$ have a reference value of $M_{I}=10^{10} \mathrm{GeV}$ in all figures. The dashed lines show the corresponding curves for the SM. The unification now occurs above $10^{16} \mathrm{GeV}$, rather than near $10^{14} \mathrm{GeV}$ as in the SM. Running is computed at two-loop order. 
To understand the nature of the unification more precisely, it is useful to look at the running of two particular combinations of the gauge couplings [26]. One combination

$$
R_{X}=\frac{1}{\sqrt{38}}\left(\frac{5}{g_{1}^{2}}-\frac{3}{g_{2}^{2}}-\frac{2}{g_{3}^{2}}\right)
$$

is shown as the solid line in the right panel of figure 1, thick blue for ISS and thin black for the SM. The other combination

$$
R_{H}=\frac{1}{\sqrt{14}}\left(\frac{3}{g_{2}^{2}}-\frac{2}{g_{3}^{2}}-\frac{1}{g_{1}^{2}}\right)
$$

is shown by the dashed lines. These are key combinations since in the minimal SU(5) model with ISS, $R_{X, H}$ vanish at energies $M_{X, H}$, the masses of the heavy SU(5) gauge bosons and the heavy color triplets that are the $\mathrm{SU}(5)$ partners of the Higgs doublets, respectively. Hence $M_{X}$ and $M_{H}$ for the reference values of the low energy particle masses can be read off directly from the right panel of figure 1 as the energy at which the solid and dashed lines cross the horizontal axis.

The deficiencies of gauge coupling unification in the SM are emphasized in this figure$M_{X}$ is two orders of magnitude below the current limit from proton decay, whereas $M_{H}$ is very far above the Planck scale. While the latter can be cured by a dimension-5 operator of the form $\left[\Sigma \mathcal{W}^{\alpha} \mathcal{W}_{\alpha}\right]_{\theta^{2}}$, additional physics is required to satisfy the experimental constraint from proton decay. With ISS, it is the combination of the gauginos, the color octet, $\Sigma_{8}$, and the weak triplet, $\Sigma_{3}$ that increase the $\mathrm{SU}(5)$ gauge boson mass to $M_{X} \simeq 7.3 \times 10^{16} \mathrm{GeV}$ at the reference point of $m_{\lambda}=M_{\Sigma_{8}}=M_{\Sigma_{3}}=10^{10} \mathrm{GeV}$. The reference point value for the colored Higgs mass is $M_{H} \simeq 5.5 \times 10^{21} \mathrm{GeV}$, still above the Planck scale, but needs only about half the correction of the SM to reduce it to of order $V \sim M_{X}$.

It is straightforward to include the effect of deviating from the reference spectrum. We then find

$$
\begin{aligned}
& \ln \left(\frac{M_{X}}{7.3 \times 10^{16} \mathrm{GeV}}\right)=-\frac{1}{6} \ln \frac{m_{\tilde{g}} m_{\tilde{w}}}{M_{I}^{2}}-\frac{1}{4} \ln \frac{m_{\Sigma_{8}} m_{\Sigma_{3}}}{M_{I}^{2}}+\delta_{X}^{I}+\delta_{X}^{U}, \\
& \ln \left(\frac{M_{H}}{5.5 \times 10^{21} \mathrm{GeV}}\right)=\frac{1}{6} \ln \frac{m_{\tilde{h}}^{4} m_{H}}{M_{I}^{5}}+\delta_{H}^{I}+\delta_{H}^{U},
\end{aligned}
$$

where

$$
\delta_{X}^{I}=-\frac{1}{8} \ln \frac{m_{\tilde{q}}^{2}}{m_{\tilde{u}} m_{\tilde{e}}}, \quad \delta_{H}^{I}=-\frac{5}{3} \ln \frac{m_{\tilde{g}}}{m_{\tilde{w}}}-\frac{5}{2} \ln \frac{m_{\Sigma_{8}}}{m_{\Sigma_{3}}}+\frac{1}{4} \ln \frac{m_{\tilde{q}}^{4}}{m_{\tilde{u}}^{3} m_{\tilde{e}}}-\frac{1}{2} \ln \frac{m_{\tilde{d}}}{m_{\tilde{l}}},
$$

parameterize squark/slepton and $\Sigma_{8 / 3}$ non-degeneracies, and the corrections $\delta_{X, H}^{U}$ contain possible terms from non-degenerate unified multiplets in non-minimal models. Here, the deviations of $M_{X, H}$ from the reference values have been calculated at the 1-loop order. We find that a deviation of the low energy spectrum from the reference mass of $10^{10} \mathrm{GeV}$ does not significantly affect the values of $M_{X}$ or $M_{H}$, unless a large deviation is considered. In particular, it is hard to bring $M_{H}$ down to of order $M_{X}$ to make the unification work by this effect alone. 
The required threshold correction, however, easily arises from higher-dimensional gauge kinetic operators. At the leading order, we may consider the dimension-5 operator of the form $\left[\Sigma \mathcal{W}^{\alpha} \mathcal{W}_{\alpha}\right]_{\theta^{2}}$, which affects $M_{H}$ significantly and can easily bring it down to $\sim 10^{17} \mathrm{GeV}$. Interestingly, this operator does not affect $M_{X}$, so that the prediction for the heavy gauge boson mass stays as in eq. (4.8). The correction to $M_{X}$ appears only at the next order from the operator of the form $\left[\left(\Sigma \mathcal{W}^{\alpha}\right)\left(\Sigma \mathcal{W}_{\alpha}\right)\right]_{\theta^{2}}$. To see these explicitly, we may replace the canonical gauge kinetic term as

$$
\operatorname{Tr}\left[\mathcal{W}^{\alpha} \mathcal{W}_{\alpha}\right] \rightarrow \operatorname{Tr}\left[\mathcal{W}^{\alpha} \mathcal{W}_{\alpha}\right]+\frac{a}{\Lambda} \operatorname{Tr}\left[\langle\Sigma\rangle \mathcal{W}^{\alpha} \mathcal{W}_{\alpha}\right]+\frac{b}{\Lambda^{2}} \operatorname{Tr}\left[\langle\Sigma\rangle \mathcal{W}^{\alpha}\right] \operatorname{Tr}\left[\langle\Sigma\rangle \mathcal{W}_{\alpha}\right]
$$

where $\Lambda$ is the UV cutoff of the unified theory. We then find that the right-hand sides of eqs. (4.8) and (4.9) receive corrections of $\left(5 \pi^{2} b / 6 g_{U}^{2}\right)\left(V^{2} / \Lambda^{2}\right)$ and $-\left(20 \pi^{2} a / \sqrt{15} g_{U}^{2}\right)(V / \Lambda)+$ $O\left(V^{2} / \Lambda^{2}\right)$, respectively, where $g_{U}$ is the unified gauge coupling at the scale $V$. In terms of $R_{X}$ and $R_{H}$, these correspond to

$$
\Delta R_{X}=\frac{5 b}{2 \sqrt{38} g_{U}^{2}} \frac{V^{2}}{\Lambda^{2}} \simeq 0.96 b \frac{V^{2}}{\Lambda^{2}}, \quad \Delta R_{H}=-\frac{\sqrt{6} a}{\sqrt{35} g_{U}^{2}} \frac{V}{\Lambda} \simeq-0.98 a \frac{V}{\Lambda} .
$$

Namely, if the solid and dashed lines in the right panel of figure 1 enter in the ranges of $\pm \Delta R_{X}$ and $\pm \Delta R_{H}$, the running of the gauge couplings can be made consistent with the $\mathrm{SU}(5)$ unification.

In the right panel of figure 1 , the corrections for $R_{X}$ and $R_{H}$ in eq. (4.12) are depicted by the dark (red) and light (pink) shaded regions, respectively, for $V / \Lambda=0.1$ with $a \in[-1,1]$ and $b \in[-1,1]$. While the second order correction $\Delta R_{X}$ is small, the first order correction $\Delta R_{H}$ is about $4 \%$ and is sufficient to lower $M_{H}$ so that it is comparable to $M_{X}$. Since this can be done with a sufficiently small value of $V / \Lambda$, unification works well in this class of models.

With the values of $M_{X, H}$ and $g_{U}$ obtained above, the rate for proton decay is expected to be small. Since the leading correction from the dimension- 5 operator in eq. (4.11) does not affect $M_{X}$, it is a relatively robust result that proton decay due to heavy gauge boson exchange is suppressed in the pure ISS models described here. However, it is possible that the rate is near the current experimental bound and within reach of future searches if the superparticle mass scale is relatively high, $\tilde{m} \sim 10^{11} \mathrm{GeV}$, because of the uncertainty of $M_{X}$ from the spectrum of intermediate scale particles; see eq. (4.8).

\subsection{ISS with TeV-scale Wino}

If the values of $m_{\mathrm{LSP}}$ allowed by scanning are less than $T_{R}$, environmental selection is likely to force a large amount of fine-tuning in $m_{\mathrm{LSP}}$, so that the amount of dark matter arising from LSP freezeout is limited. Possible selection effects include close stellar encounters, galactic disk stability [23] and baryon dilution [24]. These selection effects act on any dark matter candidate, no matter the production mechanism, so dark matter may be multicomponent, with the contribution from each component being the same order of magnitude. If the LSP is a gaugino then it must be the wino; a light bino will not annihilate efficiently and a light gluino as a significant component of dark matter is excluded. A light wino can 

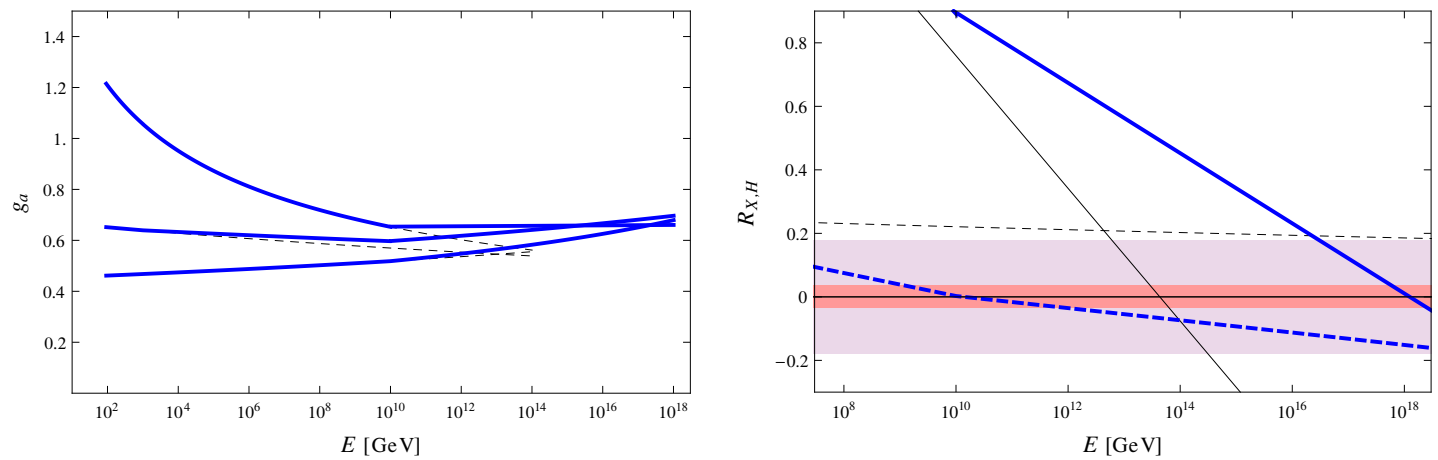

Figure 2. Intermediate scale supersymmetry breaking with TeV-scale wino. Caption as in figure 1, except both shaded (red) bands have been drawn for $V / \Lambda=0.2$.

arise from a cancellation among the various contributions to $\tilde{m}_{2}$ in eq. (4.4). If $X$ is neutral the coefficient of the $\left[X \mathcal{W}^{\alpha} \mathcal{W}_{\alpha}\right]_{\theta^{2}}$ interaction must be small to allow such a cancellation; while if $X$ is charged, so $\tilde{m}_{2}^{0}=0$, the cancellation occurs among the loop contributions in eq. (4.4).

Gauge coupling unification for ISS with the TeV-scale wino is shown in figure 2. Lowering the wino mass pushes up $M_{X}$ by over an order of magnitude since, from eq. (4.8), $M_{X} \sim 1 / m_{\tilde{w}}^{1 / 6}$, so that proton decay from $X$ exchange is greatly suppressed. On the other hand, from eq. (4.9), $M_{H} \sim m_{\tilde{w}}^{5 / 3}$ so $M_{H}$ is reduced all the way to the intermediate scale. A correction from the dimension-5 operator $\left[\Sigma \mathcal{W}^{\alpha} \mathcal{W}_{\alpha}\right]_{\theta^{2}}$ in eq. (4.11), however, can raise it back to $\sim M_{X}$, avoiding excessive proton decay from the colored Higgs triplet exchange. For $M_{H}$ to be near $M_{X}$ requires $a V / \Lambda \sim-0.2$, as shown by the shading in the right panel. Since $M_{X}$ is so large it is reasonable that the interval of validity of the unified theory is restricted.

The H.E.S.S. Collaboration is currently probing wino dark matter indirectly, via searches for monochromatic gamma lines [27]. For a NFW halo profile, wino dark matter is excluded from comprising more than about $20 \%$ of dark matter [28, 29]. However, the uncertainties from the halo profile are large, and our environmental scheme makes multi-component dark matter plausible so that the wino comprises only a part of the dark matter.

\subsection{ISS with TeV-scale Higgsino}

The remaining possibility for $m_{\mathrm{LSP}} \leq T_{R}$ is for the Higgsino mass environmentally tuned to be in the $\mathrm{TeV}$ range. The Higgsino mass parameter, $\mu$ given in eq. (4.3), undergoes tuning to the intermediate scale to allow electroweak symmetry breaking, and further tuning to the $\mathrm{TeV}$ scale allows a substantial Higgsino dark matter component. In the case that the wino and bino masses are of order $10^{10} \mathrm{GeV}$ this is excluded from direct detection probes of galactic dark matter, since in these experiments the Higgsinos act as a Dirac particle with couplings to the $Z$ boson. However, if the supersymmetry breaking field $X$ is charged, so that $\tilde{m}_{i}^{0}=0$, the remaining loop-induced gaugino masses have just the right size to render 

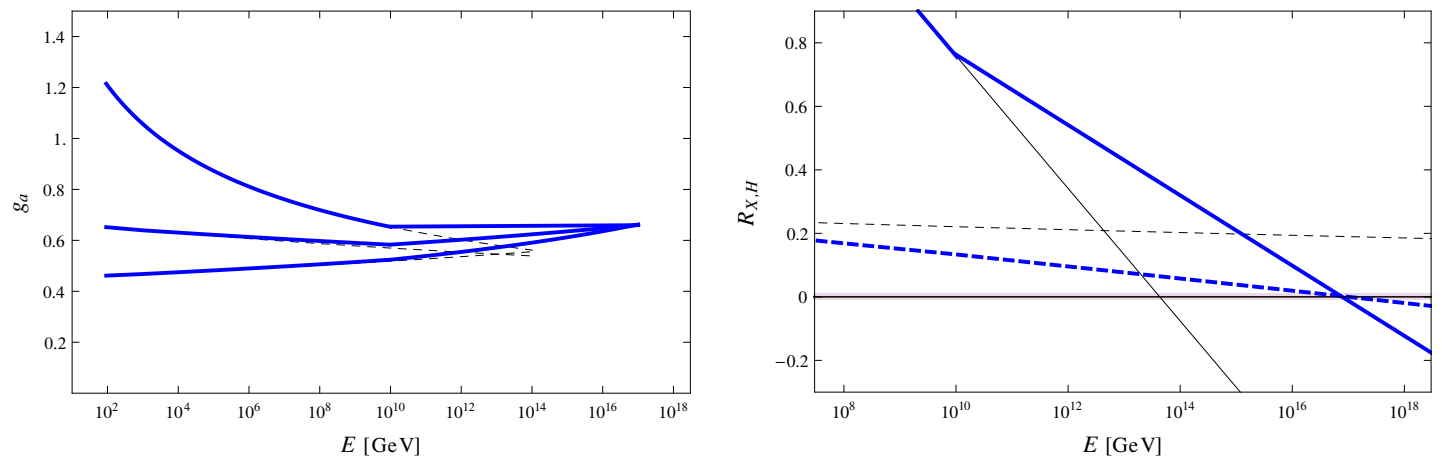

Figure 3. Intermediate scale supersymmetry breaking with TeV-scale Higgsino. Caption as in figure 1, except both shaded (red) bands have been drawn for $V / \Lambda=0.01$.

the Higgsinos as inelastic dark matter in these direct detection experiments [16], with a mass splitting between the two neutral Majorana Higgsinos of order $200 \mathrm{keV}$.

Gauge coupling unification for ISS with the TeV-scale Higgsino is shown in figure 3. Lowering the Higgsino mass does not alter $M_{X}$, so the prediction for proton decay from $X$ exchange is essentially as in the case of pure ISS discussed in section 4.1. On the other hand, since $M_{H} \sim m_{\tilde{h}}^{2 / 3}, M_{H}$ is lowered to be very close to $M_{X}$ without a threshold correction from higher-dimension operators in eq. (4.11). The accuracy of this unification is comparable to the MSSM so that, with $|a| \sim 1$, the cutoff scale $\Lambda$ could be two orders of magnitude larger than the $\mathrm{SU}(5)$-breaking $\mathrm{VEV} V$.

\section{Acknowledgments}

We recently learned that Patrick Fox, Graham Kribs, and Adam Martin are preparing a paper on Dirac gauginos where the scale of supersymmetry breaking is linked to the scale at which the SM Higgs quartic vanishes. We thank Graham Kribs for useful communications and discussions. We also thank Satoshi Shirai for discussions. This work was supported in part by the Director, Office of Science, Office of High Energy and Nuclear Physics, of the US Department of Energy under Contract DE-AC02-05CH11231 and in part by the National Science Foundation under grants PHY-0855653 and PHY-1214644.

Open Access. This article is distributed under the terms of the Creative Commons Attribution License (CC-BY 4.0), which permits any use, distribution and reproduction in any medium, provided the original author(s) and source are credited.

\section{References}

[1] H. Georgi and S.L. Glashow, Unity of all elementary particle forces, Phys. Rev. Lett. 32 (1974) 438 [InSPIRE].

[2] H. Georgi, H.R. Quinn and S. Weinberg, Hierarchy of interactions in unified gauge theories, Phys. Rev. Lett. 33 (1974) 451 [InSPIRE]. 
[3] S. Weinberg, Anthropic bound on the cosmological constant, Phys. Rev. Lett. 59 (1987) 2607 [INSPIRE].

[4] T. Banks, TCP, quantum gravity, the cosmological constant and all that..., Nucl. Phys. B 249 (1985) 332 [INSPIRE].

[5] A.D. Linde, The inflationary universe, Rept. Prog. Phys. 47 (1984) 925 [inSPIRE].

[6] V. Agrawal, S.M. Barr, J.F. Donoghue and D. Seckel, The anthropic principle and the mass scale of the standard model, Phys. Rev. D 57 (1998) 5480 [hep-ph/9707380] [INSPIRE].

[7] T. Damour and J.F. Donoghue, Constraints on the variability of quark masses from nuclear binding, Phys. Rev. D 78 (2008) 014014 [arXiv:0712.2968] [INSPIRE].

[8] B. Feldstein, L.J. Hall and T. Watari, Landscape prediction for the Higgs boson and top quark masses, Phys. Rev. D 74 (2006) 095011 [hep-ph/0608121] [INSPIRE].

[9] D. Buttazzo et al., Investigating the near-criticality of the Higgs boson, JHEP 12 (2013) 089 [arXiv: 1307.3536] [INSPIRE].

[10] A. Delgado, M. Garcia and M. Quirós, Electroweak and supersymmetry breaking from the Higgs discovery, arXiv:1312.3235 [INSPIRE].

[11] L.J. Hall and Y. Nomura, A finely-predicted Higgs boson mass from a finely-tuned weak scale, JHEP 03 (2010) 076 [arXiv:0910.2235] [INSPIRE].

[12] A. Hebecker, A.K. Knochel and T. Weigand, A shift symmetry in the Higgs sector: experimental hints and stringy realizations, JHEP 06 (2012) 093 [arXiv:1204.2551] [INSPIRE].

[13] A. Hebecker, A.K. Knochel and T. Weigand, The Higgs mass from a string-theoretic perspective, Nucl. Phys. B $\mathbf{8 7 4}$ (2013) 1 [arXiv:1304.2767] [INSPIRE].

[14] L.E. Ibáñez and I. Valenzuela, The Higgs mass as a signature of heavy SUSY, JHEP 05 (2013) 064 [arXiv: 1301.5167] [INSPIRE].

[15] Super-Kamiokande collaboration, H. Nishino et al., Search for nucleon decay into charged anti-lepton plus meson in Super-Kamiokande I and II, Phys. Rev. D 85 (2012) 112001 [arXiv:1203.4030] [INSPIRE].

[16] D. Tucker-Smith and N. Weiner, Inelastic dark matter, Phys. Rev. D 64 (2001) 043502 [hep-ph/0101138] [INSPIRE].

[17] S. Dimopoulos and H. Georgi, Softly broken supersymmetry and SU(5), Nucl. Phys. B 193 (1981) 150 [INSPIRE].

[18] N. Sakai, Naturalness in supersymmetric guts, Z. Phys. C 11 (1981) 153 [INSPIRE].

[19] G.F. Giudice and A. Masiero, A natural solution to the $\mu$ problem in supergravity theories, Phys. Lett. B 206 (1988) 480 [inSPIRE].

[20] J.A. Casas and C. Muñoz, A natural solution to the $\mu$ problem, Phys. Lett. B 306 (1993) 288 [hep-ph/9302227] [INSPIRE].

[21] L. Randall and R. Sundrum, Out of this world supersymmetry breaking, Nucl. Phys. B 557 (1999) 79 [hep-th/9810155] [InSPIRE].

[22] G.F. Giudice, M.A. Luty, H. Murayama and R. Rattazzi, Gaugino mass without singlets, JHEP 12 (1998) 027 [hep-ph/9810442] [INSPIRE]. 
[23] M. Tegmark, A. Aguirre, M. Rees and F. Wilczek, Dimensionless constants, cosmology and other dark matters, Phys. Rev. D 73 (2006) 023505 [astro-ph/0511774] [INSPIRE].

[24] R. Bousso and L. Hall, Why comparable? A multiverse explanation of the dark matter-baryon coincidence, Phys. Rev. D 88 (2013) 063503 [arXiv: 1304.6407] [InSPIRE].

[25] L.J. Hall and Y. Nomura, Spread supersymmetry, JHEP 01 (2012) 082 [arXiv:1111.4519] [INSPIRE].

[26] J. Hisano, H. Murayama and T. Yanagida, Probing GUT scale mass spectrum through precision measurements on the weak scale parameters, Phys. Rev. Lett. 69 (1992) 1014 [INSPIRE].

[27] H.E.S.S. collaboration, A. Abramowski et al., Search for photon line-like signatures from Dark Matter annihilations with H.E.S.S, Phys. Rev. Lett. 110 (2013) 041301 [arXiv: 1301.1173] [INSPIRE].

[28] T. Cohen, M. Lisanti, A. Pierce and T.R. Slatyer, Wino dark matter under siege, JCAP 10 (2013) 061 [arXiv: 1307.4082] [INSPIRE].

[29] J. Fan and M. Reece, In wino veritas? Indirect searches shed light on neutralino dark matter, JHEP 10 (2013) 124 [arXiv:1307.4400] [INSPIRE]. 